\title{
Brain Tumor Classification Based on Singular Value Decomposition
}

\author{
Nidahl K. El Abbadi ${ }^{1}$, Neamah E. Kadhim ${ }^{2}$ \\ Computer Science Department, Education College, University of Kufa, Najaf, Iraq ${ }^{1}$ \\ Computer Science Department, Science College, University of Babylon, Babylon, Iraq ${ }^{2}$
}

\begin{abstract}
Every day over 100 adults will be diagnosed with a primary brain tumor and many more will be diagnosed with a cancer. Diagnosing a specific type of brain tumor can be a complicated affair, making confirmation of its diagnosis essential. In this paper we suggested new method for detection of brain tumor based on singular value decomposition (SVD). The algorithm first trained/learned with normal brain MR images, then in the second step the algorithm become capable to classify the brain MR images into healthy and non-healthy image (that have a tumor). The algorithm is trained with 20 of normal brain MR images and tested with 50 brain MR images. The accuracy of this method was up to $97 \%$.
\end{abstract}

Keywords: MRI, brain tumor, classification, image processing, SVD.

\section{INTRODUCTION}

The medical image processing is a method of using computer algorithm in processing the medical image. The brain magnetic resonance image (MRI) classification is one of the most important field in medical image processing. The manual classification of brain MR image is a time consuming and not always gives an accurate results. Therefore there is an important for automatic classification using various technique. In these techniques the classifier first trained or learned with known data (images) that belonging to number of classes, then the classifier became capable of classifying unseen imagesaccurately into a specificclass [1].

Currently, brain tumor has become one of the main cause for increasing mortality among children and adults. It's found that the total number of people that hardship and dying from brain tumors has been increased to 300 for each year during past few decades. The primary diagnosis is frequently stated as a brain lesion. A lesion is a region in an organ or tissue that has suffered damage through disease or shock; mainly there is a different think about the brain and a part of it does not appear to like normal tissue [2].

Extra testing is commonly ordered to get a better notion of the location, size and effect of the tumor, as well as detecting any cancers in other portions of the body. It takes knowledge to be able to see specific subtle differences in MRIs. An additional opinion from knowledgeable doctor or team that regularly deals with brain tumors may alteration the preliminary diagnosis, in either tumor class or grade, and therefore alteration in the treatments. MRI only can be unsatisfied (it may not be a tumor at all), making an exhaustive examination of all of our indications (symptoms), and as soon as possible, a biopsy, vital to our diagnosis [3].

\section{RELATED WORKS}

Said Charfi [4] suggested a hybrid intelligent machine learning procedure for computer-aided detection for automatic recognition of brain tumor through MR images. The proposed method is based on the following computational procedures; the histogram dependent thresholding for image segmentation, the discrete wavelet transform for features extraction, then using PCA for reducing the dimensionality of the extracted features, and the feed forward back-propagation neural network to classify inputs into normal or abnormal. The accuracy of the classification on both training and test images is $90 \%$ which was considerably good.

J. Umamaheswari and Dr. G. Radhamani [5] proposed a hybrid approach for medical image classification. This approach consists of feature extraction and classification. The classification consists of Multi Linear Discriminate Analysis (MLDA) and Support Vector Machine (SVM).

Classification is done on the base of parameter extracted by Gray Level Co-occurrence Matrix (GLCM) and histogram texture feature extraction method.The efficiency of the method has been assessed using the following measures:Accuracy, Sensitivity and Specificity, the proposed method get $94 \%, 87 \%$ and $92 \%$ respectively.

Rosy Kumari [6] suggested method based on Grey-Level Co-occurrence matrix (GLCM) and Support Vector Machine (SVM). The suggested method involves of two steps: feature extraction and classification. In first step,the features are extracted from images using GLCM.

In the next step, the features that are extracted fed as input to SVM classifier. Theimages are classified into normal and abnormal along with variety of disease depending upon features. 


\section{THEORY OF SINGULAR VALUE DECOMPOSITION (SVD)}

Singular Value Decomposition (SVD) has lately occurred as a new hypothesis for processing different kinds of images. SVD is a smart algebraic transform for processing the image applications [8].

The SVD is an optimal matrices decomposition in the least square sense that it packets the determined signal energy into a few number of coefficients. Singular value decomposition (SVD) is an effective and firm methods to splitting the system to a set of independent components, each component has own energy contribution. Singular value decomposition (SVD) is a technique that used in numerical to diagonalizable matrixes in mathematical analysis [9]. SVD is a pretty numerical transform for image processing, because of its unending advantages, such as extreme energy packing which is usually used in compression to manipulate the image in the base of two distinctive subspaces noise and data sub spaces, which is usually uses in the image noise filtering and also used in watermarking application [10].

These applications exploit important properties of the SVD. Also it is usually used in solving least squares problem, computing pseudo- inverse of a matrix and multivariate analysis. SVD is strong and trustworthy orthogonal matrix decomposition procedures, which is due to its conceptual and stability reasons becoming further general in signal processing. SVD has the capability to get used in the variations in local statistics of an image [8]. A digital Image $\mathrm{X}$ of size $\mathrm{M} * \mathrm{~N}$, with $\mathrm{M} \geq \mathrm{N}$, can be represented by its SVD as follows: [8]

$$
\begin{gathered}
{[\mathbf{X}]_{M}=\mathrm{M}[\mathbf{U}][\mathbf{S}] \mathrm{M}\left[\mathbf{V}^{\mathrm{N}}\right]^{\mathrm{T}} \mathrm{N}} \\
\mathrm{U}=\left[\mathrm{u}_{1}, \mathrm{u}_{2}, \ldots \mathrm{u}_{\mathrm{m}}\right], \\
\mathrm{S}=\left[\begin{array}{llll}
\sigma_{1} & & \\
& \sigma_{2} & & \\
& & \mathrm{O} & \\
& & \sigma_{\mathrm{n}}
\end{array}\right]
\end{gathered}
$$

Where $\mathrm{U}$ is a $(\mathrm{M} * \mathrm{M})$ orthogonal matrices, $\mathrm{V}$ is an $(\mathrm{N} *$ $\mathrm{N})$ orthogonal matrices, and $\mathrm{S}$ is an $(\mathrm{M} * \mathrm{~N})$ matrices with the elements of diagonal represents the singular values(SV), Si of X. Using the T subscript to indicate the transpose of the matrices. The matrices columns of $U$ is called the left singular vectors, and the matrices columns of $\mathrm{V}$ are called the right singular vectors.

Each singular value (SV) identifies the image luminance while the corresponding couple of singular vectors (SCs) specifies the image geometry. $\mathrm{U}$ and $\mathrm{V}$ are unitary orthogonal matrices (the sum of squares to columns is unity and all of these columns are uncorrelated) and $\mathrm{S}$ is a diagonal matrix (only the leading diagonal has non-zero values) of decreasing singular values [11].

\section{METHODOLOGY OF THE PROPOSED METHOD}

SVD is a method that can transform the image data into multiplication of three matrices USV. The diagonal elements of matrix Srepresent the singular values that hold the most important information of the original image MR images, these values can be used to represent the image.

The using of singular values of such refactoring (applied SVD to image) allows us to represent the image with a smaller set of values, which can preserve most important features of the original image, these values called singular values SV. These values represented in diagonal matrices $S$ that have the following properties: for $i=1$ to $n, \sigma_{i}$ are singular values that:

$$
\begin{aligned}
\sigma_{1>} & =\sigma_{2>}=\ldots . .>=\sigma_{\mathrm{r}>0} \\
\sigma_{\mathrm{r}+1} & =\sigma_{\mathrm{r}+2}=\ldots . .=\sigma_{\mathrm{n}}=0
\end{aligned}
$$

These singular values are ordered in the diagonal and have values greater than zero and all other values are zero in the matrices S. That means the first no. of these SVs are have the most important features of the image and can use to recognize the different MRI brain images.

The proposed method consist of two stages, first the algorithm trained/learned with normal brain MR images to compute the base value from singular values. In the second stage the algorithm classify the brain MR images to normal or abnormal (with tumor) depending on singular values. The group of normal MRI brain images are treated as a set of known normal brain MRI as vectors in a space, called "normal space". Then singular value decomposition SVD computed for each vector of normal space (for each normal MR image into space), the resulted singular values extracted into vectors, for each vector compute the norm and these values put into a vector, at the end calculate the mean value of this vector to be the base value. To classify the brain MR images, the new image first loaded and compute SVD to it, compute the singular values SV that treated to produce the norm value called NewV that compare with the base value $\mathrm{BaseV}$ if the difference less than specific threshold then the brain MRI is Normal (not has a tumor), else if the difference lager than threshold the brain MRI is Abnormal and has a tumor.

The two stages of SVD classification method is detailed in the following steps:

\section{* $\quad$ First stage : (learning/trained stage)}

Step1: Load the MRI brain images from database Normal brain MR images.

Step2: Resize the MR image.

Step3:Convert the image to the gray level image.(if the image is RGB Image).

Step4: For each image in database Compute the Singular Value Decomposition [U S V].

Step5: Extract the diagonal values from $\mathrm{S}$ matrix that have singular Values(SV).

Step6: Compute the norm for each SV in step 4 and put the values invector called SVNorm. 
Step7: Compute Mean value of SV Norm to be the base value called Base V.

* Second stage: classification stage Step1: Load the brain MR image to be classify Step2: Resize the image.

Step3: Convert the image to the gray level image.

Step4: Compute SVD of new image (U, S, and V)

Step5: Extract the diagonal element of S (SV)

Step6: Compute Norm to the SV called NewV

Step7: Classify to Normal or Abnormal

Difference $=\operatorname{abs}($ BaseV $-\mathrm{NewV})$

If (Difference) $<=$ THR

Then the Brain MRI is Normal

Else Brain MRI is Abnormal

Where the THR is a threshold that computed accurately by experiments.

The algorithm trained with 20 normal brain MR image and tested with 10 normal and 50 abnormal MR images. The proposed method reduce the time and effort needed for classification the brain MR images into healthy (normal) or not healthy (has a tumor), figure 1 show the flowchart of proposed algorithm.

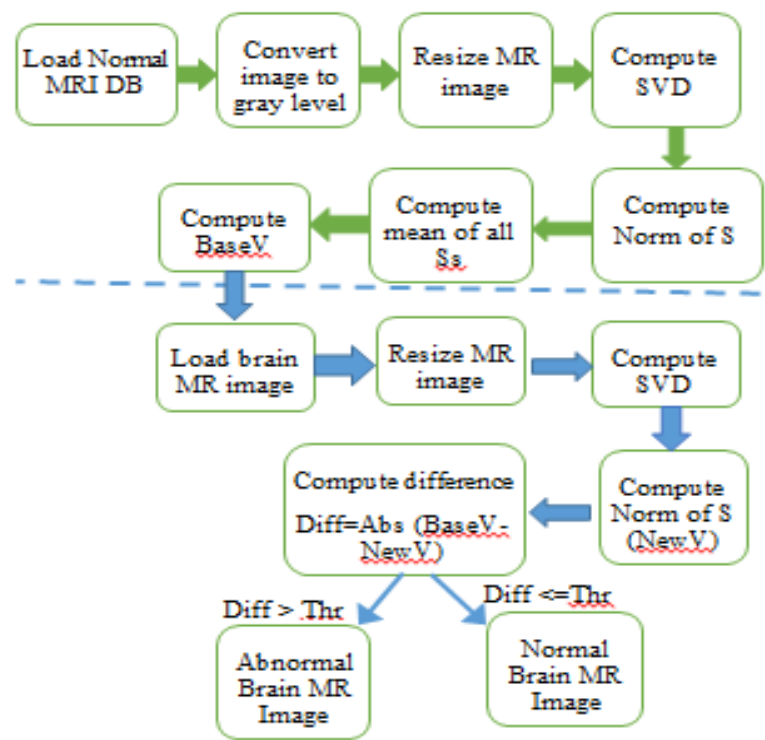

Fig. 1 Flowchart of the proposed method

\section{BRAIN MR IMAGE DATA SET}

The brain MR images (brain tumor and normal MRI) have been obtained from different medical centers (MR Imaging of Neoplastic Central Nervous System and the whole brain tumor Atlas that belong to the Harvard medical school) in JPEG format. The MRI images have been obtained from these centers of different kinds of brain MR normal images and brain tumor MR images like Sarcoma, Anaplastic astrocytoma and Meningioma. Figure 2 represent the examples of the brain MR images database, where A, B, C and D represent example of normal, Anaplastic astrocytoma, Sarcoma and Meningioma.
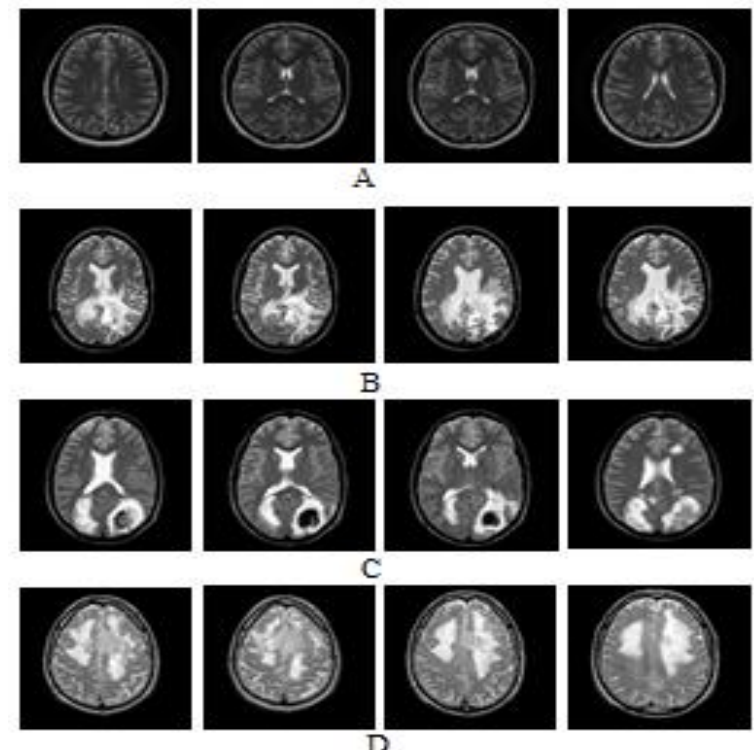

Fig. 2. Example of brain MR images database

\section{RESULT AND DISCUSSION}

Twentynormal brain MR image are used to training the classifier, also ten normal brain MR images and fifty brain tumor MR image (from different classes) are used in testing the proposed classification method. In the first stage (training stage), the Basev value is calculated and the result was 19.0659. In the second stage the $\mathrm{NewV}$ has been computed for the new images. The selected threshold was 5.0 which selected carefully from experiments on normal and abnormal MR images. Figure 3 show example of some results from implementing the proposed classification methods which show three instances of abnormal classification and one for normal classification. Table1 show the NewV and difference (Diff) values of 12 example of brain MR images from the database. This table show the proposed method failed to classify only two brain MR images which showed in the figure4.
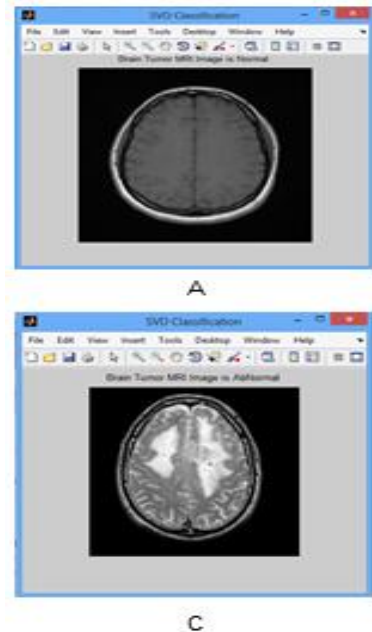

Fig. 3.The implementation of SVD classificationA as

normal classification and $\mathrm{B}, \mathrm{C}$ and $\mathrm{D}$ as Abnormal classification
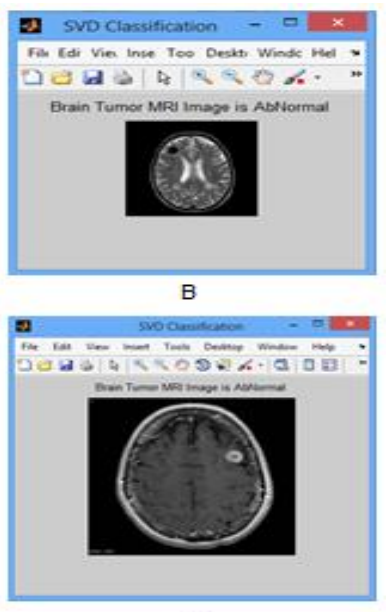
Vol. 5, Issue 8, August 2016

Table1. Testing results of proposed SVD method classification on MRI examples

\begin{tabular}{|l|l|l|l|l|l|}
\hline No. & Image & Class & NewV & Difference & Classification \\
\hline 1 & Img1 & Normal & 15.7 & 3.2 & Normal \\
\hline 2 & Img2 & Normal & 16.86 & 2.19 & Normal \\
\hline 3 & Img3 & Normal & 15.18 & 3.8 & Normal \\
\hline 4 & Img4 & Normal & 16.4 & 2.5 & Normal \\
\hline 5 & Img5 & Abnormal & 29.08 & 10.2 & Abnormal \\
\hline 6 & Img6 & Abnormal & 34.56 & 15.5 & Abnormal \\
\hline 7 & Img7 & Abnormal & 36.79 & 17.7 & Abnormal \\
\hline 8 & Img8 & Abnormal & 35.8 & 16.8 & Abnormal \\
\hline 9 & Img9 & Abnormal & 28.3 & 9.2 & Abnormal \\
\hline 10 & Img10 & Abnormal & 30.8 & 11.7 & Abnormal \\
\hline 11 & Img11 & Normal & 26.08 & 7 & Abnormal \\
\hline 12 & Img12 & Abnormal & 21.5 & 2.5 & Normal \\
\hline
\end{tabular}
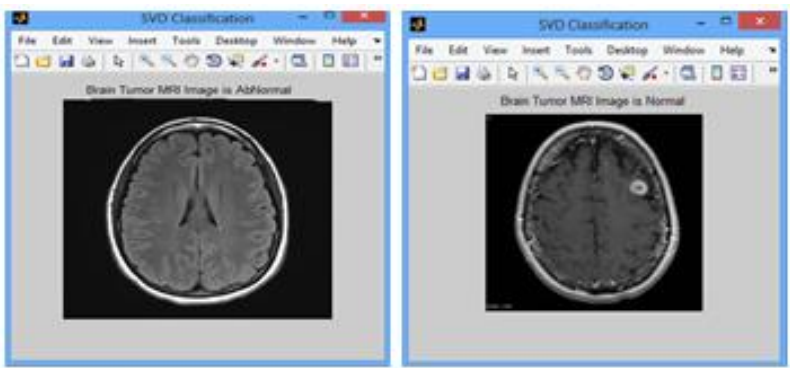

Fig. 4. Misclassification brain MR images by the proposed method

The evaluation of the proposed method have been calculatedin term of predictive values, there are four predictive values: true positive value (TP), true negative value $(\mathrm{TN})$, false negative value $(\mathrm{FN})$ and false positive value (FP). These values are used to calculate the performance of the proposed method results which applied tobrain MR images by sensitivity, specificity and accuracy of the system. Table 2 showed the predictive values as the behavior of the proposed method, also in the table 3 the proposed SVD classification results is compared with the previous works.

The sensitivity: This is the probability of positive result giventhat

Sensitivity $=[\mathrm{TP} /(\mathrm{TP}+\mathrm{FN})]^{*} 100$.

Sensitivity $=[9 /(9+1)]^{*} 100=90 \%$

The specificity: This is the probability of negative result

Specificity $=[\mathrm{TN} /(\mathrm{FP}+\mathrm{TN})]^{*} 100$

Specificity $=[49 /(1+49)]^{*} 100=98 \%$

Accuracy: Accuracy is how close a measured value is to the

actual (true) value.

Accuracy $=$ No. of correct diagnostic/No. of all data .. (3) $=[(\Sigma$ True positive $+\Sigma$ True negative $) / \Sigma$ Total population]*100
Accuracy $=[(9+49) / 60] * 100=96.66$

Table2. Brain MR Image rates used in training and testing

\begin{tabular}{|c|c|c|c|}
\hline $\begin{array}{c}\text { Total no } \\
\text { of Images }\end{array}$ & $\begin{array}{c}\text { No of Normal } \\
\text { Images in Training }\end{array}$ & \multicolumn{2}{|c|}{$\begin{array}{l}\text { No. of Images in } \\
\text { Testing }\end{array}$} \\
\cline { 2 - 4 } & Normal MRI & Normal & Abnormal \\
\hline 80 & 20 & 10 & 50 \\
\hline
\end{tabular}

Table3. Performance of proposed SVD classifier compared with other works

\begin{tabular}{|l|c|c|c|c|c|c|c|}
\hline \multicolumn{1}{|c|}{ Method } & TP & TN & FP & FN & $\begin{array}{c}\text { Sensitivity } \\
\%\end{array}$ & $\begin{array}{c}\text { Specificity } \\
\%\end{array}$ & $\begin{array}{c}\text { Accuracy } \\
\%\end{array}$ \\
\hline Said Charfi[4] & - & - & - & - & - & - & 90 \\
\hline J.\& D. G. [5] & - & - & - & - & 94 & 87 & 92 \\
\hline Rosy Kumari[6] & 33 & 31 & 37 & 0 & 100 & 82 & 90 \\
\hline Proposed & 9 & 49 & 1 & 1 & 90 & 98 & 96.6 \\
\hline
\end{tabular}

\section{CONCLUSION}

The proposed method using SVD as a classifier for classification the brain MR images produce a good classification accuracy when compared to other classifiers. The proposed singular value decomposition method proved that it is sensitive to the changes into the MR images and produce good result in classification of the human brain MR images. The sensitivity, specificity and accuracy is also developed.The future work is to increase the SVD classification accuracy by increasing the training database.

\section{REFERENCES}

[1] Girja Sahu and Lalit Kumar P. Bhaiya, " A survey paper based on classification of MRI brain images using soft computing technique", International Journal of Emerging Technology and Advanced Engineering (ISSN 2250-2459, ISO 9001:2008 Certified Journal, Volume 4, Issue 12, December 2014.

[2] A. Mustaqeem, et al., "An Efficient Brain Tumor Detection Algorithm Using Watershed \&Thresholding Based Segmentation", International Journal of Image, Graphics and Signal Processing, Vol.4, No.10, 2012, pp34-39.

[3] Brain Tumor DiagnosisApproved by the Cancer.Net Editorial Board, (C) 2005-2016 American Society of Clinical Oncology (ASCO).

http://www.cancer.net/cancer-types/brain-tumor/diagnosis

[4] Said Charfi, Redouan Lahmyed and Lalitha Rangarajan, "A novel Approach for Brain Tumor Detection Using Neural Network", IMPACT: International Journal of Research in Engineering \& Technology (IMPACT: IJRET) ISSN(E): 2321-8843; Vol. 2, Issue 7, Jul 2014, 93-104.

[5] J. Umamaheswari and Dr. G. Radhamani, "A Hybrid Approach for Classification of DICOM Image", ISSN: 2221-0741 Vol. 1, No. 8,364-369, 2011.

[6] Rosy Kumari, "SVM Classification an Approach on Detecting Abnormality in Brain MRI Images", International Journal of Engineering Research and Applications, Vol. 3, Issue 4, Jul-Aug 2013, pp.1686-1690.

[7] Nitish Zulpe and Vrushsen Pawar, "GLCM Textural Features for Brain Tumor Classification", International Journal of Computer 
Science Issues, Vol. 9, Issue 3, No 3, May 2012 ISSN (Online): 1694-0814.

[8] Rowayda A. Sadek, " SVD Based Image Processing Applications: State of The Art, Contributions and Research Challenges", International Journal of Advanced Computer Science and Applications, Vol. 3, No. 7, 2012.

[9] T. Konda, Y. Nakamura, "A new algorithm for singular value decomposition and its parallelization", Parallel Computing 35(6):331-344,2009, doi:10.1016/j.parco.2009.02.001.

[10] Dobrovolny M. Šilar Z., Černy M. "Asymmetric Image Compressionfor Embedded Devices based on Singular Value Decomposition”,IEEE Applied Electronics Pilsen, 2011.

[11] Satish Kumar Singh, Shishir Kumar, "A Framework to Design Novel SVD Based Color Image Compression", EMS '09 proceeding of the 2009 Third UKSim European Symposium on Computer Modeling and Simulation, pp 235-240, doi:10.1109/EMS.2009.100.

\section{BIOGRAPHIES}

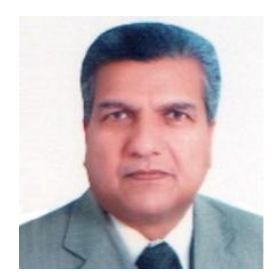

Nidhal El Abbadi, received B.Sc. in Chemical Engineering, B.Sc. in computer science, M.Sc. and Ph.D. in computer science, worked in industry and many universities, reviewer for a number of international journals, has many published papers and five published books (Programming with Pascal, $\mathrm{C}++$ from beginning to OOP, Data structures in simple language, computer graphics, effect of modern technology on talent)and chapter in Handbook of Research on Threat Detection and Countermeasures in Network Security book, his research interests are in image processing, security, and steganography, He's Professor in Computer Science in the University of Kufa - Najaf, IRAQ.

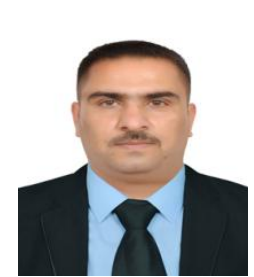

Neamah .E Kadhim received B.Sc. in Computer Science, M.Sc. in Computer Science from college of Science, Baghdad University. Now Ph.D. student in college of science at the Babylon University through research stage. His interest in image processing, steganography and windows programming. He's Assistance lecture in Computer Science in the University of Baghdad, IRAQ. 